\title{
Variations in PET/MRI Operations: Results from an International Survey Among 39 Active Sites
}

\author{
Wolfgang Peter Fendler ${ }^{1}$, Johannes Czernin ${ }^{1}$, Ken Herrmann ${ }^{1}$, and Thomas Beyer ${ }^{2}$ \\ ${ }^{1}$ Department of Molecular and Medical Pharmacology, David Geffen School of Medicine at UCLA, Los Angeles, California; and \\ ${ }^{2}$ Centre of Medical Physics and Biomedical Engineering, QIMP, Medical University of Vienna, Vienna, Austria
}

\begin{abstract}
Information has been collected from PET/MRI operational sites to identify its present and future applications. This may help to focus discussions on common interests of the PET/MRI community. Methods: A web-based survey of PET/MRI users was conducted from June to October 2015. The survey was composed of 26 questions related to the PET/MRI center, present use and imaging protocols, and perspectives on key applications. Results: Responses were collected from 39 international sites that operated PET/MRI for a median of $30 \mathrm{mo}$ (range, 2-62 mo). Most installations were located in public institutions with an academic focus $(n=26,67 \%)$. Systems were primarily operated by nuclear medicine departments $(n=13,33 \%)$, jointly by nuclear medicine and radiology ( $n=11,28 \%)$, and radiology only $(n=10,26 \%)$. PET/MRI operation was equally focused on clinic routine and research $(47 \%$ vs. $45 \%$ of sites, respectively). Sites reported a strong focus on oncology ( $76 \%$ of research and $88 \%$ of clinical applications). Other applications included neurology ( $9 \%$ clinical, $12 \%$ research) and cardiology ( $3 \%$ clinical, $6 \%$ research). Perceived superiority over $\mathrm{PET} / \mathrm{CT}$ was identified as the strongest driver for clinical adoption. Over half the operators expect PET/MRI to excel in clinical routine within 3-5 y. Emerging key applications for future PET/MRI use were cardiovascular disease and imaging of inflammation. Conclusion: An international survey of early PET/MR adopters reveals a mixed use of this combined imaging modality, with a focus on oncology. The future of PET/MRI is seen in expanded application for oncology and neurology, but also cardiovascular disease and inflammation.
\end{abstract}

Key Words: PET/MRI; survey; imaging protocols

J Nucl Med 2016; 57:2016-2021

DOI: 10.2967/jnumed.116.174169

\section{O} multaneous preclinical PET/MR imaging was proposed in 1997 by Cherry's group at University of California, Los Angeles (1). Since then, the concept of combining PET and MRI within a single examination has been refined technologically and methodologically, first in small animals and, as of 2006, in humans (2). This is different from PET/CT that initially addressed a clinical

Received Feb. 12, 2016; revision accepted Jun. 30, 2016.

For correspondence or reprints contact: Ken Herrmann, University of California at Los Angeles, Ahmanson Translational Imaging Division, 10833 Le Conte Ave., 200 Medical Plaza, Ste. B114-61, Los Angeles, CA 90095-7370.

E-mail: kherrmann@mednet.ucla.edu

Published online Aug. 11, 2016.

COPYRIGHT (C) 2016 by the Society of Nuclear Medicine and Molecular Imaging, Inc.

DOI: $10.2967 /$ jnumed.116.174169 need (3) before being adapted for small-animal imaging $(4,5)$. $\mathrm{PET} / \mathrm{CT}$ is a tremendous success story, with more than 5,000 systems installed worldwide today. Within the first 3 y after market introduction in 2001, more than 500 PET/CT systems had been installed. By comparison, the adoption of PET/MRI has been slow. After the introduction of the first prototype (designed for brain imaging) in 2007 (6) and the installation of 5 more units worldwide, the first commercially available whole-body PET/MRI system was introduced in 2011 (7). Two fully integrated PET/MRI systems were introduced in 2011 (8) and 2014 (9). Five years after its introduction, only around 70 PET/MRI systems have been placed worldwide.

Advantages of PET/MRI integration may include the nearsimultaneous acquisition of molecular images and high-resolution anatomic images of high soft-tissue contrast as well as the exploitation of functional MR imaging parameters to aid the development of diagnostic and intermediate endpoint biomarkers. Thus, much has been published about the potential of combined PET/MRI. Yet little clinical evidence is available to support an added value of this modality in comparison to other, established imaging modalities $(10,11)$. Several expert panels have commented on the potential of PET/MR imaging for clinical specific applications, such as brain or cardiac imaging, as well as for research applications that are based on multiparametric anatometabolic imaging (12-16). Although PET/MRI is an innovative technology, it has not yet found its place in clinical practice. Current users are early adopters, and their PET/MRI use patterns may provide insights into current and future key applications. The current survey of international PET/MRI users was conducted to record and document such current use patterns in clinical routine and research.

\section{MATERIALS AND METHODS}

\section{Concept}

A survey of international PET/MRI users was designed in February 2015 to collect data on existing PET/MRI operations worldwide and to review PET/MRI protocol variations. The idea for this data collection originated from discussions during the recent PET/MRI workshop in Tübingen (16) and a subsequent review of the international survey on PET/CT operations conducted a few years earlier (17). As in this previous study, we decided to benefit from the easy, rapid, and wide-spread distribution of web-based questionnaires. Similar to our previous study, we prepared our survey in line with recent recommendations on the structure of surveys that-in theory-help to maximize response rates; this included a personal introductory statement, the offer to make results public, the use of simple headers and textual representation of response categories, and the provision of a relatively short deadline including multiple reminders. 


\section{Survey}

The electronic questionnaire consisted of 26 questions (supplemental materials [supplemental materials are available at http://jnm. snmjournals.org]) that could be answered in about $20 \mathrm{~min}$. Questions, developed by imaging experts with extensive experience in PET/CT and PET/MR imaging, addressed 3 issues: the operation of the PET/ MRI centers $(n=9)$, current applications and imaging protocols $(n=14)$, and perspectives on future key applications $(n=3)$. Question types were dichotomous, single choice, multiple choice, rating scales, or open ended for number, text comment, or date. The survey was composed in English using an internet-based platform (SurveyMonkey.com). Invitation to the survey was sent in June 2015 to the complete e-mail listing of all active whole-body PET/MRI physicians or researchers ( 1 key user per site) at the time. This list of 69 active sites was composed of feedback we solicited from participants of the most recent PET/MRI expert panel meeting in Tübingen 2015 (16) and the 3 vendors of whole-body PET/MR imaging systems: Philips Healthcare, Siemens Healthcare, and GE Healthcare. Three reminders were mailed to all nonresponding recipients between June 2015 and August 2015. The survey was closed on November 1, 2015.

\section{Statistical Analysis}

Responses were collected anonymously and tabulated for each question. We report total number of responses per answer option, proportion of responses per respective answer option in percent, rank of answer options, or median (range) for quantitative response. Free text response for key applications (questions 23 and 24) was summarized by field of application into 5 categories (Cardiovascular, Pediatric, Other, Neurology, Oncology) with respective subcategories.

\section{RESULTS}

Thirty-nine responses were collected from 69 active PET/MRI users between June and October 2015. All responses were satisfactory in quality and quantity, and therefore the overall response rate was $57 \%$.

\section{Demographics: PET/MRI Center (Questions 1-9)}

The 39 responding sites were located in Europe (62\%), North America (23\%), and Asia Pacific (16\%) (Fig. 1A). Responding sites operated PET/MR systems from Siemens Healthcare (77\%), Philips Healthcare (13\%), and GE Healthcare (10\%) (Fig. 1B), which corresponded well to the known market shares of these companies at time of the survey $(72 \%, 14 \%$, and $14 \%$, respectively).

The number of active PET/MRI sites grew continuously from 2010, with an average number of 8 sites becoming operational each year (Fig. 1C). Responding sites had operated PET/MRI for a median of 30 mo (range, 2-62 mo), and 23 of 39 (59\%) sites had PET/MRI experience for $2 \mathrm{y}$ or more.

Table 1 lists funding and clinical responsibility separate for private and public institutions as well as for different operating departments. Most PET/MRI systems (26/39, 67\%) were placed in public institutions with an academic focus. Systems were operated by nuclear medicine $(n=13,33 \%)$, jointly between departments $(n=11,28 \%)$, or by radiology $(n=10,26 \%)$. Other operators $(n=5,13 \%)$ were departments of radiation therapy $(n=2)$ and research institutions $(n=3)$.

Clinical scans were reported jointly by nuclear medicine + radiology in private $(7 / 10,70 \%)$ and public $(23 / 29,79 \%)$ institutions. Interdisciplinary reporting was performed in almost all nuclear medicine and jointly operated departments (92\% and 91\%);

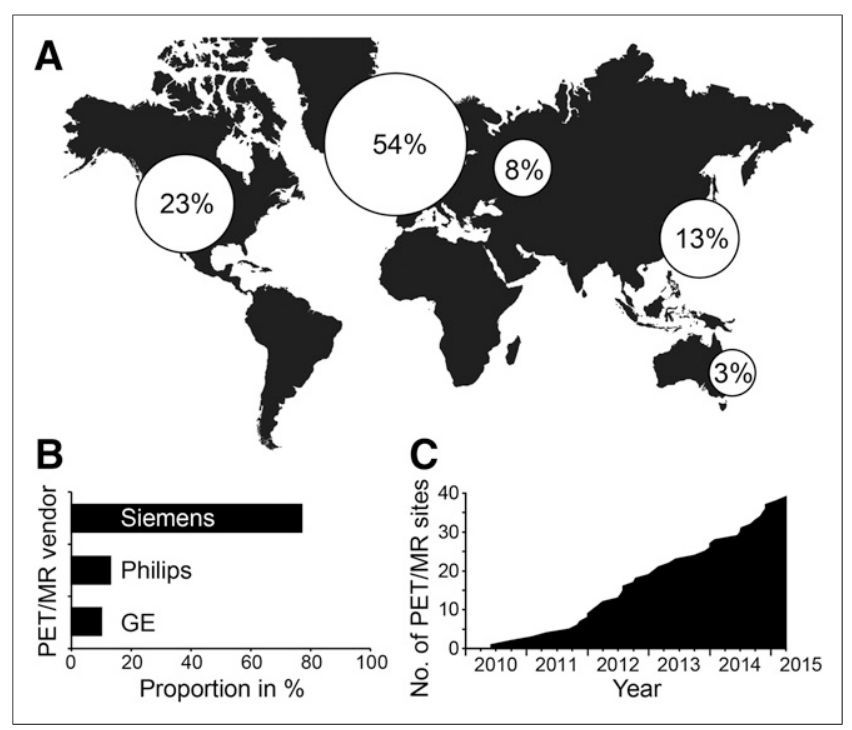

FIGURE 1. Survey of 39 PET/MRI sites by location (A), vendor (B), and first year of operation (C). PET/MRI location was categorized into (from left to right) North America, Western Europe, Eastern Europe, Asia, and Australia.

however, $40 \%$ of reports from PET/MRI clinics operated by radiology were provided only by radiologists.

Hospital, private, and research funds were almost equally regarded as a leading source of financing for the PET/MRI device ( $26 \%, 28 \%$, and $28 \%$, respectively). Governments provided main funds in $18 \%$ of sites. Nine of $21(43 \%)$ devices in Western Europe were primarily funded by research grants versus $11 \%$ of devices elsewhere. A median of 10 employees participated in PET/MRI operation, including 4 physicians, 2 technicians, 2 physicists, and 2 administrative staff. The median number of staff was lower for public $(n=9)$ than private $(n=15)$ institutions and for joint, nuclear medicine, or other departments $(n=10)$ than radiology departments $(n=13)$. The median number of employees in institutions with PET/MRI interpretation by radiologists or nuclear medicine physicians only $(n=11)$ was similar to that of the entire group $(n=10)$.

\section{Present Applications (Questions 10-13 and 15-16)}

Figure 2 demonstrates the most prevalent applications as reported by the survey participants. Forty-seven percent and $45 \%$ of PET/MRI operations focused on clinical and research, respectively. The main applications were oncology (76\% of research and $88 \%$ of clinical applications) and neurology (12\% and 9\%, respectively). Several centers operated PET/MRI for clinical $(13 \%)$ or research purposes (19\%) only.

Within both clinical and research oncologic applications, participants ranked prostate cancer, brain tumors, and head and neck cancers highest. Few sites ranked cardiac ( $n=3$ sites) and other applications highest ( $n=2$ sites).

In total, 44,706 patients underwent PET/MRI at the responding sites, which corresponds to an average throughput of about 8 (maximum, 38) patients per week. This average number, derived from the total number of patients scanned divided by the number of weeks of operation for each site, however, was lower than the present throughput reported by the sites at 12 (maximum, 35) patients per week. 
TABLE 1

PET/MRI Financial Resources As Well As Clinical Responsibility Separated by Provider

\begin{tabular}{|c|c|c|c|c|c|c|c|}
\hline Category & All & Private & Public & Joint & Nuc & Rad & Other \\
\hline$n$ & 39 & 10 & 29 & 11 & 13 & 10 & 5 \\
\hline \multicolumn{8}{|l|}{ Funded by } \\
\hline Hospital & $10(26 \%)$ & $3(30 \%)$ & 7 (24\%) & $3(27 \%)$ & $3(23 \%)$ & $4(40 \%)$ & 0 \\
\hline Government & 7 (18\%) & 0 & 7 (24\%) & $3(27 \%)$ & 0 & $1(10 \%)$ & $3(60 \%)$ \\
\hline Private & $11(28 \%)$ & $5(50 \%)$ & $6(21 \%)$ & $3(27 \%)$ & $5(38 \%)$ & $3(30 \%)$ & 0 \\
\hline Res grant & $11(28 \%)$ & $2(20 \%)$ & 9 (31\%) & $2(18 \%)$ & 5 (38\%) & $2(20 \%)$ & $2(40 \%)$ \\
\hline \multicolumn{8}{|l|}{ Reported by } \\
\hline Joint & 30 (77\%) & 7 (70\%) & 23 (79\%) & 10 (91\%) & 12 (92\%) & $5(50 \%)$ & $3(60 \%)$ \\
\hline Nuc only & $1(3 \%)$ & $1(10 \%)$ & 0 & 0 & 1 (8\%) & 0 & 0 \\
\hline Res only & $3(8 \%)$ & 0 & $3(10 \%)$ & 0 & 0 & $1(10 \%)$ & $2(40 \%)$ \\
\hline Rad only & 5 (13\%) & 2 (20\%) & $3(10 \%)$ & $1(9 \%)$ & 0 & $4(40 \%)$ & 0 \\
\hline MRI-only scans & 25 (64\%) & 8 (80\%) & 17 (59\%) & $7(64 \%)$ & 7 (54\%) & 7 (70\%) & $4(80 \%)$ \\
\hline
\end{tabular}

Joint = Joint Nuclear Medicine/Radiology Department; Nuc = Nuclear Medicine Department; Rad = Radiology Department; Res $=$ research.

Absolute numbers, with percentages in parentheses, are given.

\section{Variations in Imaging Protocols (Questions 14 and 17-22)}

Imaging protocols vary widely across active PET/MRI sites (Table 2). Because no PET/MRI standard or guidelines have been published, survey questions regarding typical imaging protocols were phrased more generically. Typical cardiology and oncology imaging protocols were reported to take a median of $60 \mathrm{~min}$, with

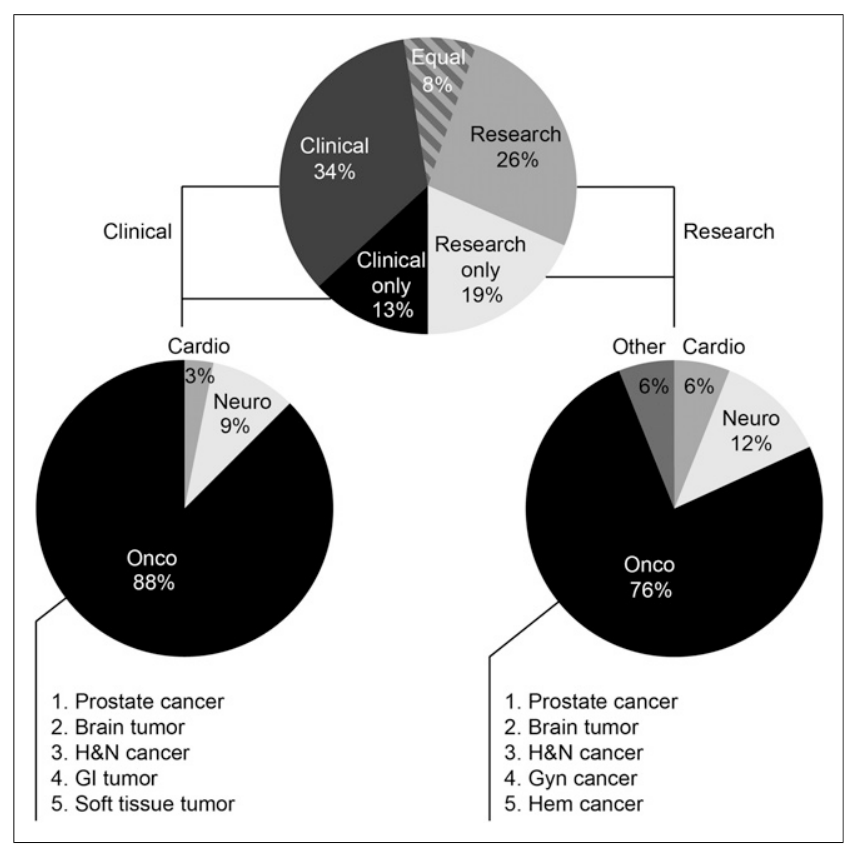

FIGURE 2. Present clinical and research applications. Most prevalent clinical and research applications have been categorized into cardiology (Cardio), neurology (Neuro), other, or oncology (Onco). Oncology was further subdivided by tumor type. $\mathrm{Gl}=$ gastrointestinal; Gyn = gynecologic; $\mathrm{H} \& \mathrm{~N}=$ head \& neck; Hem = hematologic. a 30-min minimum and 100- to 120-min maximum. A typical neurology imaging protocol was somewhat shorter, with a reported median of 45 min (range, 10-120 min).

The most frequently used PET probe across all categories and applications was ${ }^{18} \mathrm{~F}-\mathrm{FDG} .{ }^{13} \mathrm{~N}_{-} \mathrm{NH}_{3}$ (ranked 2), ${ }^{18} \mathrm{~F}$-FET (ranked 3 ), and prostate-specific membrane antigen ligands (ranked 3) were also used frequently.

MR protocols were heterogeneous, with a certain degree of overlap (Supplemental Table 1). All reported neurology MRI protocols included diffusion-weighted imaging or fluid attenuation inversion recovery in addition to $\mathrm{T} 1$ and $\mathrm{T} 2$ acquisition. MR protocols for oncology imaging frequently included Dixon (5/8), diffusion-weighted imaging (4/8), and volumetric interpolated breath-hold examination (3/8) sequences. About two thirds of the sites $(25 / 39,64 \%)$, foremost those from the private sector $(80 \%)$ and those within radiology, radiation therapy, or research departments (70\%-80\%), performed MRIonly protocols in addition to PET/MR imaging (Table 1). Most patients would, in the user's perspective $(25 / 39,64 \%)$, have undergone MRI and PET/CT in the case that PET/MRI was not available.

\section{Present and Future Key Applications (Questions 23 and 24)}

Participants named up to $3 \mathrm{key}$ applications for present and future use of PET/MRI (Fig. 3). Oncology $(n=24)$ and neurology $(n=13)$ were most frequently mentioned for present key application, followed by pediatric $(n=4)$ and other $(n=7)$ imaging. General oncology and prostate cancer were most prevalent oncology indications; general neurology and dementia imaging were the most prevalent neurology indications.

None of the participants listed cardiovascular disease at present; however, 7 participants included cardiovascular imaging, for example, for diagnosis of cardiac fibrosis, atherosclerosis, or vasculitis, as future key applications of PET/MRI. Furthermore, imaging inflammation was considered a new application within 
TABLE 2

Scan Time and Tracer Separated by Field of Application

\begin{tabular}{|c|c|c|c|}
\hline Parameter & Cardiology & Neurology & Oncology \\
\hline Typical duration of scan (min) & $60(30-120)$ & $45(10-120)$ & $60(30-100)$ \\
\hline \multicolumn{4}{|l|}{ Top 3 tracer used } \\
\hline 1 & ${ }^{18} \mathrm{~F}-\mathrm{FDG}$ & ${ }^{18} \mathrm{~F}-\mathrm{FDG}$ & ${ }^{18} \mathrm{~F}-\mathrm{FDG}$ \\
\hline 2 & ${ }^{13} \mathrm{~N}-\mathrm{NH}_{3}$ & Other & Other \\
\hline 3 & Other & ${ }^{18} \mathrm{~F}-\mathrm{FET}$ & PSMA ligands \\
\hline
\end{tabular}

the next years by 5 participants. Future pediatric, oncology, and neurology PET/MRI had fewer mentions. Further votes for future key applications were cast for musculoskeletal disease $(n=1)$, lymphoma $(n=2)$, soft-tissue $(n=2)$, and liver $(n=1)$ tumors.

\section{Clinical Adoption (Questions 25 and 26)}

Table 3 summarizes the user's perspective on clinical adoption of PET/MRI. Most operators expected increased use in the midterm $(\sim 3-5 \mathrm{y} ; 28 / 39,72 \%)$. Superiority over PET/CT and unique clinical information were ranked highest among the anticipated drivers for adoption, followed by cost, combined functional MRI and PET, and patient throughput ranked last.

\section{DISCUSSION}

The aim of this survey was to determine how PET/MRI is used worldwide. This survey follows in its design other surveys on the adoption of PET/CT (17) and SPECT/CT (18). A decade after the introduction of PET/CT more than 5,000 systems from 6 different vendors have been installed worldwide $(17,19)$. At the time of this survey, about $5 \mathrm{y}$ into commercialization of whole-body PET/MRI, fewer than 80 systems from 3 vendors have been installed at near linear growth rate. The current survey helps to identify strengths and weaknesses of PET/MRI and may provide insights into the reasons for its slow adoption.

First, the duration of a typical PET/MRI protocol (45-60 min) was about 2 times longer and weekly throughput (8-12 patients) about 5 times lower than in typical PET/CT operations, despite

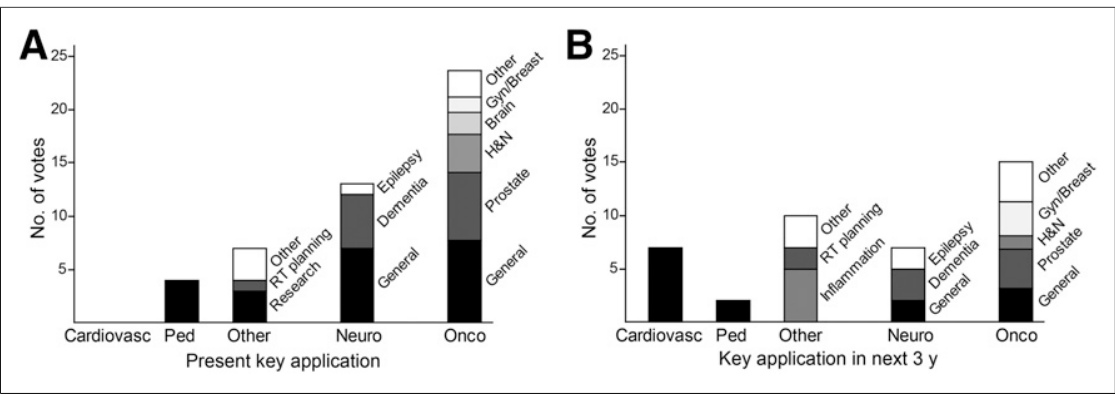

FIGURE 3. Present (A) and future (B) key applications. Participants listed up to 3 key applications for present and future PET/MRI use. Cardiovasc = cardiovascular; Gyn = gynecologic; H\&N = head and neck; Neuro $=$ neurologic; Onco $=$ oncologic; Ped $=$ pediatric; $\mathrm{RT}=$ radiation therapy. similar number of staff used (17). This difference likely reflects a higher complexity of the MRI versus CT component in terms of active operator involvement and integration with PET. Further, most PET/MRI systems were installed in Western Europe after considerable research funding for these sites. The low patient throughput and the amount of public and research funding raises questions about the financial viability of privately operated systems.

Second, protocols are not well defined, mostly because of varying MRI protocols. Members of the Tübingen PET/MRI expert panels expressed their clear intent to harmonize future PET/MRI operation (15). However, imaging protocols, especially those of the MRI component, remain highly variable as shown in the current survey. Variation of MRI protocols is apparently needed to enable further innovation at this early stage of PET/MRI operation. However, in a long-term perspective, harmonization of specific protocols may be of use in demonstrating clinical utility needed to accelerate PET/MRI adoption. The reported selection of PET tracers, however, revealed 1 clear similarity among the participating sites: ${ }^{18} \mathrm{~F}-\mathrm{FDG}$ remains the most important tracer for PET/MRI. Prostate-specific membrane antigen ligands were ranked among the top 3 tracers for oncology, even before choline analogs or somatostatin ligands. Such prominent position of a relatively new radioligand further underlines the dominant role of prostate cancer proposed both for present and for future use of PET/MRI in cancer patients. ${ }^{18} \mathrm{~F}-\mathrm{FET}$ was ranked no. 3 for brain applications, even before compounds for neurodegenerative disease, thus indicating an important role of PET/MRI for neurooncology. ${ }^{13} \mathrm{~N}-\mathrm{NH}_{3}$ ranked no. 2 for cardiac applications, however, at an overall low volume of cardiovascular examinations.

Third, the interpretation of PET/MR images is more challenging than that of PET/CT studies. This was reflected in our survey by the fact that interpretation was jointly performed by radiologists and nuclear medicine physicians at more than $80 \%$ of PET/MRI sites versus only approximately $40 \%$ of PET/CT sites in our previous survey (17).

PET/MRI was performed equally often for clinic and research. Oncology was the dominant application of present clinical and research use $(88 \%$ and $76 \%$, respectively). Cancers that were imaged frequently with stand-alone MRI before the availability of PET/MRI (i.e., patients with 
TABLE 3

Timeline for Clinical Adoption of PET/MRI

\begin{tabular}{lccc}
\hline \multicolumn{1}{c}{ Parameter } & All & Private & Public \\
\hline$n \quad$ & 39 & 10 & 29 \\
$\begin{array}{c}\text { In how many years } \\
\text { will PET/MRI excel } \\
\text { in clinical routine? }\end{array}$ & & & \\
1 & $4(10 \%)$ & 0 & $4(14 \%)$ \\
3 & $13(33 \%)$ & $5(50 \%)$ & $8(28 \%)$ \\
5 & $15(38 \%)$ & $2(20 \%)$ & $13(45 \%)$ \\
\hline 10 & $6(15 \%)$ & $3(30 \%)$ & $3(10 \%)$ \\
$>10$ & $1(3 \%)$ & 0 & $1(3 \%)$ \\
\hline
\end{tabular}

prostate cancer, brain tumors, and head and neck cancer) were listed as the top 3 indications. The focus on these tumor entities, for which MRI may be considered an anatomic modality-ofchoice, is reasonable if cost-effectiveness is demonstrated and patient comfort is acceptable. However, this ranking of indications also underlines a current lack of definite new indications for fully integrated $(8,9)$ PET/MR imaging.

The survey revealed a low current or future proportion of neurologic and cardiovascular PET/MRI examinations. This is surprising as these indications were frequently named as key future applications $(2,20)$. Cardiovascular disease was not mentioned as a key present indication by any of the participants; pediatric imaging was named as a key application by only 4 of 39 sites and was even less frequently mentioned as a key future indication. This is surprising, given the frequently publicized radiation concerns of the pediatric medical community $(21,22)$. However, imaging of inflammation and cardiovascular diseases was voted first and second most anticipated future indication.

In general, the current survey suggests that both current and future applications might evolve around oncologic entities and questions. Given the high use of PET/CT and its acceptable costs in cancer (23), it remains questionable whether this will result in dramatic increases in PET/MRI use in cancer patients. However, PET/MRI is still at the level of early adoption, with several unsolved technical, regulatory, and funding issues. Use patterns of the current respondents might therefore not be representative for the PET/MRI community of the future.

One potential advantage of near-simultaneous data acquisition is the exact temporal and spatial correlation of morphologic, functional, and metabolic information. Exact fusion is needed to combine complementary PET and MRI information of a dynamic system, such as moving organs or transient pathologies. Drzezga et al. proposed a complementary value of brain metabolism (PET) and regional perfusion (MRI) and pointed to a clinical advantage through their simultaneous acquisition (24). However, the clinical benefit of near-simultaneous versus sequential acquisition of such biomarkers has not yet been confirmed (16). PET/MRI applications for which near-simultaneous image acquisitions were proposed as advantageous such as neurodegenerative diseases (24) contributed to less than $10 \%$ of the clinical PET/MRI workload.

Despite the lack of novel future applications, more than $50 \%$ of respondents predict a profound clinical impact of PET/MRI within
$5 \mathrm{y}$. Interestingly, more than $15 \%$ of respondents predict an impact of PET/MRI only after the next decade. Despite the apparent lack of key applications and the obvious need for future development, PET/MRI users expressed their confidence in the potential of this technology by ranking superiority over PET/CT among the top drivers for clinical adoption. Evidence for such superiority, however, is not yet available (11).

\section{CONCLUSION}

This survey among early adopters of combined PET/MR imaging systems demonstrates a mixed perspective on the current and futureuse case scenarios of this imaging modality, with a focus on oncology, particularly with prostate, brain, and head and neck malignancies. Today's PET/MRI users predict evidence for diagnostic benefits over PET/CT as one of the drivers for clinical transition.

\section{DISCLOSURE}

The costs of publication of this article were defrayed in part by the payment of page charges. Therefore, and solely to indicate this fact, this article is hereby marked "advertisement" in accordance with 18 USC section 1734. Johannes Czernin is cofounder of Momentum Biosciences, Sofie Biosciences, and Trethera Therapeutics, Los Angeles, USA. Thomas Beyer is cofounder and owner of cmi-experts $\mathrm{GmbH}$, Basel, Switzerland, and academic collaborator with Siemens Healthcare. Ken Herrmann is a consultant of Sofie Biosciences, Los Angeles, USA. No other potential conflict of interest relevant to this article was reported.

\section{ACKNOWLEDGMENT}

We thank the participants of this survey for their support.

\section{REFERENCES}

1. Shao Y, Cherry SR, Farahani K, et al. Simultaneous PET and MR imaging. Phys Med Biol. 1997;42:1965-1970.

2. Schlemmer HP, Pichler BJ, Schmand M, et al. Simultaneous MR/PET imaging of the human brain: feasibility study. Radiology. 2008;248:1028-1035.

3. Beyer T, Townsend DW, Brun T, et al. A combined PET/CT scanner for clinical oncology. J Nucl Med. 2000;41:1369-1379.

4. Szanda I, Mackewn J, Patay G, et al. National Electrical Manufacturers Association NU-4 performance evaluation of the PET component of the NanoPET/CT preclinical PET/CT scanner. J Nucl Med. 2011;52:1741-1747.

5. Magota K, Kubo N, Kuge Y, Nishijima K, Zhao S, Tamaki N. Performance characterization of the Inveon preclinical small-animal PET/SPECT/CT system for multimodality imaging. Eur J Nucl Med Mol Imaging. 2011;38:742-752.

6. Schmand M, Burbar Z, Corbeil J, et al. BrainPET: first human tomograph for simultaneous (functional) PET and MR imaging [abstract]. J Nucl Med. 2007;48 (suppl 2):45P.

7. Zaidi H, Ojha N, Morich M, et al. Design and performance evaluation of a wholebody Ingenuity TF PET-MRI system. Phys Med Biol. 2011;56:3091-3106.

8. Delso G, Furst S, Jakoby B, et al. Performance measurements of the Siemens mMR integrated whole-body PET/MR scanner. J Nucl Med. 2011;52:1914-1922.

9. Delso G, Khalighi M, Hofbauer M, Porto M, Veit-Haibach P, von Schulthess G. Preliminary evaluation of image quality in a new clinical ToF-PET/MR scanner. EJNMMI Phys. 2014;1:A41.

10. Czernin J, Herrmann K. The potential of PET/MRI imaging in oncology: a comment to a summary report of the first PET/MRI Workshop in Tuebingen in 2012. Mol Imaging Biol. 2013;15:372-373.

11. Spick C, Herrmann K, Czernin J. ${ }^{18} \mathrm{~F}-\mathrm{FDG}$ PET/CT and PET/MRI perform equally well in cancer patients: evidence from studies in more than 2300 patients. J Nucl Med. 2016;57:420-430. 
12. Wahl RL, Quint LE, Cieslak RD, Aisen AM, Koeppe RA, Meyer CR. "Anatometabolic" tumor imaging: fusion of FDG PET with CT or MRI to localize foci of increased activity. J Nucl Med. 1993;34:1190-1197.

13. Bailey DL, Antoch G, Bartenstein P, et al. Combined PET/MR: the real work has just started - summary report of the Third International Workshop on PET/MR imaging; February 17-21, 2014, Tubingen, Germany. Mol Imaging Biol. 2015; 17:297-312.

14. Bailey DL, Barthel H, Beuthin-Baumann B, et al. Combined PET/MR: where are we now? Summary report of the Second International Workshop on PET/MR Imaging; April 8-12, 2013, Tubingen, Germany. Mol Imaging Biol. 2014;16:295-310.

15. Bailey DL, Barthel H, Beyer T, et al. Summary report of the First International Workshop on PET/MR Imaging, March 19-23, 2012, Tubingen, Germany. Mol Imaging Biol. 2013;15:361-371.

16. Bailey DL, Pichler BJ, Guckel B, et al. Combined PET/MRI: multi-modality multi-parametric imaging is here-summary report of the 4th International Workshop on PET/MR imaging; February 23-27, 2015, Tubingen, Germany. Mol Imaging Biol. 2015;17:595-608.

17. Beyer T, Czernin J, Freudenberg LS. Variations in clinical PET/CT operations: results of an international survey of active PET/CT users. J Nucl Med. 2011;52:303-310.
18. Wieder H, Freudenberg LS, Czernin J, Navar BN, Israel O, Beyer T. Variations of clinical SPECT/CT operations: an international survey. Nuklearmedizin. 2012;51:154-160.

19. Beyer T, Townsend DW, Czernin J, Freudenberg LS. The future of hybrid imagingpart 2: PET/CT. Insights Imaging. 2011;2:225-234.

20. Marsden PK, Strul D, Keevil SF, Williams SC, Cash D. Simultaneous PET and NMR. Br J Radiol. 2002;75:S53-S59.

21. Gatidis S, Schmidt H, Gucke B, et al. Comprehensive oncologic imaging in infants and preschool children with substantially reduced radiation exposure using combined simultaneous ${ }^{18} \mathrm{~F}$-fluorodeoxyglucose positron emission tomography/magnetic resonance imaging: a direct comparison to ${ }^{18} \mathrm{~F}$-fluorodeoxyglucose positron emission tomography/computed tomography. Invest Radiol. 2016; 51:7-14.

22. Purz S, Sabri O, Viehweger A, et al. Potential pediatric applications of PET/MR. J Nucl Med. 2014;55:32S-39S.

23. Yang Y, Czernin J. Contribution of imaging to cancer care costs. J Nucl Med. 2011;52(suppl 2):86S-92S.

24. Drzezga A, Barthel H, Minoshima S, Sabri O. Potential clinical Applications of PET/MR imaging in neurodegenerative diseases. J Nucl Med. 2014; $55: 47 \mathrm{~S}-55 \mathrm{~S}$ 